\title{
Was nehmen wir nicht (ausreichend) wahr?
}

\author{
Jann Arends ${ }^{a}$ Freerk T. Baumann $^{b}$ Hans Lampe $^{c}$ Anna Paul $^{d}$ \\ ${ }^{a}$ Abt. Gastroenterologie und Ernährung, Klinik für Tumorbiologie, Freiburg, \\ ${ }^{b}$ Abteilung für molekulare und zelluläre Sportmedizin, Institut für Kreislaufforschung und Sportmedizin, Deutsche Sporthochschule Köln, \\ ${ }^{c}$ Klinik für Innere Medizin III, Universitätsklinikum Rostock, \\ ${ }^{d}$ Klinik für Naturheilkunde und Integrative Medizin, Kliniken Essen-Mitte, Knappschafts-Krankenhaus, Essen, Deutschland
}

\section{Sport und Bewegung im Therapiekonzept}

Verantwortlicher Autor: Freerk T. Baumann, Köln

Bis vor etwa 20 Jahren wurde jegliche sportliche Betätigung in Zusammenhang mit Krebserkrankungen kritisch beurteilt. Es wurde befürchtet, dass körperliche Bewegung die Metastasierung von Tumoren begünstigt oder sich nachteilig auf die medizinischen Therapieergebnisse auswirkt. Nicht selten wurden Forderungen erhoben wie «Ein halbes Jahr Bewegungsverbot nach Chemotherapie». In der Folge litten Patienten nicht nur unter ihrer Erkrankung und den therapieassoziierten Nebenwirkungen, sondern häufig auch unter einer Bewegungsmangelsymptomatik. Die nachteiligen Effekte übermäBiger Bettruhe sind hinlänglich bekannt. Bereits bei 7-tägiger Inaktivität verringert sich die Muskelkraft deutlich, das Herzvolumen sinkt, die Sauerstoffaufnahme im Blut nimmt ab und das Immunsystem ist geschwächt. Ohnehin ist das Aktivitätsniveau von Patienten, die eine Krebstherapie absolviert haben, um 20-30\% im Vergleich zur Situation vor der Erkrankung reduziert - weshalb sowohl in der Akutphase als auch in der Nachsorge ein besonderes Augenmerk auf den Erhalt bzw. die Wiederherstellung der körperlichen Leistungsfähigkeit gelegt werden sollte. Dies wirkt sich auch positiv auf die Psyche und das soziale Umfeld des Patienten aus und wird damit zum Maßstab für eine bessere Lebensqualität.

Denn ganz im Gegensatz zu den früheren Annahmen wirkt sich körperliche Aktivität bei Krebspatienten in vielfacher Hinsicht vorteilhaft aus. Dies gilt für objektiv messbare Parameter wie die physische Leistungsfähigkeit und die Schmerzverarbeitung ebenso wie für die allgemeine Befindlichkeit. So kann Bewegung das Selbstwertgefühl stärken, Ängste mindern und die Motivation steigern. Seit Anfang der 1980erJahre erste Untersuchungen zu den Effekten körperlicher Aktivität bei Krebserkrankungen publiziert wurden [1], ist das Interesse an diesem Forschungsgebiet rasch gewachsen.
Mittlerweile liegen etwa 2000 aussagekräftige Studien dazu vor. Das Thema ist längst in der Praxis angekommen und wird auch umgesetzt; so gibt es in Deutschland schon über 1000 Krebssportgruppen.

\section{Sport und Rezidivrisiko}

Auch wenn mit sportlicher Betätigung bzw. körperlicher Aktivierung vordringlich die Lebensqualität gebessert werden soll, stellt sich auch die Frage nach einer möglichen Beeinflussung des Krankheitsverlaufes. So konnten Holmes et al. [2] zeigen, dass regelmäßige, moderate körperliche Aktivität (3bis 5-mal wöchentlich jeweils 1 Stunde) das Rezidivrisiko nach Brustkrebs senkt. Mit körperlicher Aktivität ist auch jegliche Form der Bewegung im Alltag gemeint, also etwa auch Treppensteigen, Einkaufengehen usw. Dieser Hinweis ist nach unserer Erfahrung hilfreich, um Patienten zu mehr Bewegung zu motivieren, zumal bei übergewichtigen Patienten nach Studienlage das Bewegungsprogramm noch aufgestockt werden sollte (5-7 Stunden moderate körperliche Aktivität pro Woche bei Patienten mit einem Body-Mass-Index $(\mathrm{BMI})>25)$. Auch bei Prostatakrebs scheint sich körperliche Aktivität (3-mal pro Woche 1 Stunde intensives Training) günstig auszuwirken: Kenfield et al. [3] werteten über 2700 Patientendaten aus und ermitteln für sportlich aktive Männer eine Reduktion der Gesamtmortalität um 49\% und der prostataspezifischen Mortalität um 61\%.

\section{Sport während der Therapie}

Beispielhaft wird hier auf die günstigen Effekte eines Krafttrainings während der Chemotherapie von Patientinnen mit Brustkrebs eingegangen. Frauen, die regelmäßig trainierten, litten bereits ab der ersten Chemotherapiewoche signifikant weniger unter Fatigue und entwickelten mehr Kraft in beiden Armen, so das Ergebnis entsprechender noch laufender Untersuchungen in Köln. Dass sich auch mit einem Ausdauer-

\section{KARGER}

Fax +497614520714

Information@Karger.de

www.karger.com (c) 2012 S. Karger GmbH, Freiburg

0378-584X/12/3517-0012\$38.00/0

Accessible online at:

www.karger.com/onk 


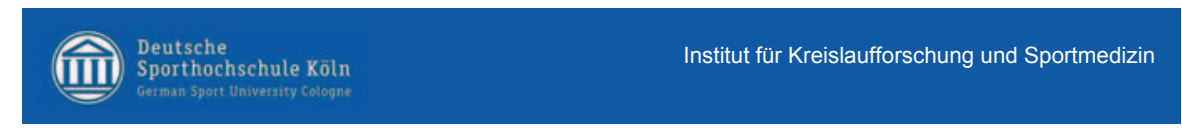

Fatigue und Lebensqualität

Abb. 1. Effekte eines Ausdauertrainings auf Fatigue und Lebensqualität (modifiziert nach $[7,8])$.

\begin{tabular}{|l|c|c|c|c|}
\hline Trainingsgruppe & Aufnahme $(\overline{\mathrm{x}} \pm \mathrm{S})$ & Entlassung $(\overline{\mathrm{x}} \pm \mathrm{s})$ & Entwicklung (\%) & Signifikanz \\
\hline Lebensqualitat & $63,7 \pm 19,7$ & $68,6 \pm 11,2$ & $+7,7$ & n. s. \\
\hline Fatigue & $41,8 \pm 25,3$ & $43,8 \pm 22,7$ & $+4,8$ & n. s. \\
\hline
\end{tabular}

Übersicht über ausgewählte Score-Ergebnisse der Trainingsgruppe ( $N=17)$ des EORTC QLQ-C30 (1-100) von Tag-6 bis zur Entlassung) im prä-post-Vergleich (t-Test; Mittelwerte, Standardabweichungen (s), Tag -6 bis zur Entlassung) im prä-post-Vergleich (t-T)
Irrtumswahrscheinlichkeit $\left.(p) ;{ }^{*}=p \leq 5 \% ;{ }^{* *}=p \leq 1 \%\right)$

\begin{tabular}{|l|c|c|c|c|}
\hline Kontrollgruppe & Aufnahme $(\overline{\mathrm{x}} \pm \mathrm{S})$ & Entlassung $(\overline{\mathrm{x}} \pm \mathrm{S})$ & Entwicklung $(\%)$ & Signifikanz \\
\hline Lebensqualitat & $62,5 \pm 23,9$ & $56,3 \pm 17,6$ & $-9,9$ & n. s. \\
\hline Fatigue & $36,1 \pm 24,5$ & $52,8 \pm 27,1$ & $+46,3$ & $\mathrm{p}=0,046$ \\
\hline
\end{tabular}

Übersicht über ausgewählte Score-Ergebnisse der Kontrollgruppe ( $N=16)$ des EORTC QLQ-C30 (1-100) von Tag -6 bis zur Entlassung) im prä-post-Vergleich (t-Test; Mittelwerte, Standardabweichungen (s), Irrtumswahrscheinlichkeit $(p) ;{ }^{*}=p \leq 5 \%$; $\left.{ }^{* *}=p \leq 1 \%\right)$ Baumann et al. 2010 und 2011 training viel erreichen lässt, zeigt eine weitere Untersuchung unter Einschluss von 66 Patienten mit Prostatakarzinom [4]. Sie nahmen während der Bestrahlung 3-mal wöchentlich an einem Walking-Programm teil und konnten damit die Gehstrecke trotz der belastenden Strahlentherapie steigern. Eine ähnlich konzipierte Studie [5] wies für diese Patientengruppe nach, dass sich mit Ausdauertraining auch die Fatigue-Symptomatik während einer Bestrahlung reduzieren lässt. Mit einem Krafttraining wiederum lässt sich die Lebensqualität von Männern mit Prostatakarzinom unter Hormontherapie bessern [6], um eine weitere Studie aus diesem Themenfeld anzuführen. Selbst Patienten, die sich einer allogenen Stammzelltransplantation unterziehen müssen und entsprechend belastet sind, können kurze Trainingseinheiten umsetzen und davon profitieren. Wir konnten dies bei Patienten nachweisen, die während ihres gesamten stationären Aufenthaltes 2-mal pro Tag für jeweils 10-20 Minuten mit dem Fahrradergometer trainierten. Die körperliche Belastbarkeit dieser Patienten war gegenüber einer untrainierten Vergleichsgruppe signifikant besser. Das Training leistete auch einen Beitrag zum Erhalt der Lebensqualität (mit einem Fragebogen der European Organisation for Research and Treatment of Cancer (EORTC) erfasst; Abb. 1) und reduzierte tendenziell die therapiebegleitende Fatigue [7,8].

\section{Sport in der Nachsorge und Rehabilitation}

Sportangebote nehmen in der Nachsorge einen immer größeren Raum ein und sind fester Bestandteil von Rehabilitationsmaßnahmen. Manche Patienten brauchen dabei speziell auf sie zugeschnittene Angebote. So profitieren etwa Frauen mit Lymphödem von Kraftübungen, mit denen sich bei konsequentem Training über einen Zeitraum von 52 Wochen das
Risiko für Exazerbationen signifikant senken lässt [9]. Intensiv trainierende Patienten sollten dabei unbedingt die empfohlenen Regenerationszeiten von jeweils 1-2 Tagen nach jeder anstrengenden Trainingseinheit einhalten. Während dieser Zeit laufen physiologische und immunologische Vorgänge $a b$, die für den Erhalt/Aufbau der Leistungsfähigkeit wichtig sind. So wurde unter anderem gezeigt, dass die Aktivität der natürlichen Killerzellen nach dem Training nur langsam wieder ansteigt und erst nach etwa 1 Tag wieder das Ausgangsniveau erreicht [10].

Gezielte Übungen sind auch hilfreich, wenn Patienten im Zuge einer organbezogenen Krebstherapie eine Inkontinenz entwickeln. Mit einem Beckenbodentraining konnte innerhalb von 1 Jahr die Kontinenzrate deutlich gesteigert werden, besonders bei Studienteilnehmern, die ein therapeutengestütztes Training erhalten hatten [11]. Bemerkenswert sind auch die Ergebnisse einer Studie, deren Teilnehmer bereits 1 Monat vor einer anstehenden Prostata-Operation mit dem Beckenbodentraining begonnen hatten [12]. Mit einer Kontinenzrate von fast $60 \%$ (3 Monate postoperativ) erreichten die Frühstarter ein deutlich besseres Ergebnis als die Gruppe, die erst postoperativ mit dem Training angefangen hatte.

\section{Sport und Bewegung soll Freude bereiten}

Das Thema Sport und körperliche Aktivität [13] lässt sich ohne viel Aufwand in die ärztliche Sprechstunde integrieren und wird von vielen Patienten mit großem Interesse verfolgt. Natürlich kann man auch bei Sport etwas falsch machen, jedoch gibt es nichts Risikoreicheres als unbegründete Schonung bzw. Bewegungsmangel. Wir erleben immer wieder, dass zunächst sehr vorsichtige Patienten nach entsprechender Anleitung oder Teilnahme an Kursen ihre sportlichen Vorhaben umsetzen. 
Was empfehle ich der Patientin?

Abb. 2. Allgemeine Empfehlungen zu Sport und Bewegung.

\begin{tabular}{|l|}
\hline Arzt (Onkologie) \\
> allg. Bewegungsempfehlung \\
$>$ Aufklärung betreiben \\
$>$ Sicherheit schenken \\
$>$ Unsicherheit nehmen \\
- „Es gibt nichts Risikoreicheres \\
als körperliche Inaktivität“ \\
- „Tun Sie das, was Ihnen gut tut“ \\
- „Vertrauen Sie Ihrem Körper“ \\
- „Beginnen Sie mit leichten \\
Intensitäten und steigern Sie \\
langsam“ \\
- „Die Bewegungsform/Sportart \\
wählen, an der Sie Freude haben“
\end{tabular}

Arzt,

Sportwissenschaftler (Sportmedizin)

spez. Trainings- und

Therapiesteuerung

$>$ Leistungsdiagnostik

$>$ Training vor dem

Hintergrund der

- Krebsentität,

- Medikation,

- Nebenwirkungen,

- individuellen Ressourcen
Dass dabei eine Sportart bzw. Bewegungsform gewählt wird, die Freude macht, sollte selbstverständlich sein. Bei einer sportmedizinischen Beratung (Abb. 2) werden zusätzlich Hintergrundinformationen vermittelt, die interessierten Patienten eine Hilfe sein können. In diesem Zusammenhang sei auf die Stellungnahme einer amerikanischen Expertengruppe verwiesen [14]. Sie kommt zu dem Schluss, dass sportliche Aktivität während und nach einer Krebstherapie zu einer Steigerung der Fitness und Muskelkraft führt und die Lebensqualität bessert. Außerdem kann damit eine Fatigue vermieden oder verringert werden, insbesondere bei Patienten mit Brustkrebs, Prostatakrebs und hämatologischen Neoplasien.

\section{Aspekte der Ernährung bei Krebserkrankungen Verantwortlicher Autor: Jann Arends, Freiburg i.Br.}

Ernährung aus klassisch medizinischer Sicht ist Ernährungsund Stoffwechselmedizin. Bei genauer Betrachtung nimmt Ernährung jedoch einen viel breiteren Raum im Leben jedes Einzelnen ein. Und dies hat wesentliche Bedeutung für viele an Krebs Erkrankte, da mehrere oder alle Bedeutungsaspekte der Ernährung durch die Erkrankung beeinträchtigt oder sogar aufgehoben sein können und häufig sind. Ohne Ernährung ist Leben nicht denkbar, dennoch spielen Ernährungsfragen in der onkologischen Forschung und auch in der onkologischen Klinik bisher nur eine marginale Rolle. Es bleibt zu hoffen, dass sich diese Situation mittelfristig ändert.

Neben dem grundlegenden Einfluss auf biochemisch-metabolische Abläufe hat die Nahrungsaufnahme relevante emotionale und soziale Bedeutung und sie dient als wichtiges Feld für die kognitive Planung und Kontrolle des eigenen individu- ellen Verhaltens. Emotionale Basis, soziale Einbindung und Kontrolle des eigenen Körpers können so bei Ernährungsstörungen mit betroffen und gefährdet sein.

\section{Die emotionale Komponente}

Genuss und Ekel als gegensätzliche Pole beschreiben die emotionale Skala der durch Nahrungsaufnahme ausgelösten Empfindungen. Diese uralte Koppelung von emotionalen Empfindungen an die Prozesse der Nahrungssuche, -aufnahme und -verarbeitung dient von jeher der Verankerung und Sicherung zuträglicher, gesunder Verhaltensweisen. Genussreiches Essen und anschließende Wohligkeit sind nur möglich in stressarmer Umgebung und vermitteln einen Schutz vor Stress, Unsicherheit und Ängsten. Der emotionale Gewinn durch Nahrungsaufnahme unterstützt im Idealfall die Konzentrations- und Kommunikationsfähigkeit [15]. Er fördert Verständnis, Akzeptanz und Compliance - auch für medizinische Argumente - sowie Leistungsfähigkeit, Lebensqualität und möglicherweise die Immunkompetenz. Genau diese Aspekte sind jedoch in Gefahr, wenn Geruchs- oder Geschmacksstörungen, Appetitverlust, Übelkeit und Erbrechen, Völlegefühl und nahrungsassoziierte Schmerzen oder Diarrhö permanent anhalten und die Nahrungsaufnahme nicht nur unattraktiv, sondern unerträglich machen. Es spricht viel dafür, Ansätze zu fördern, die diese Aspekte differenziert analysieren und in therapeutische Konzepte umsetzen [16].

Der mögliche Genuss des Essens kann auch durch - von Ärzten, Heilpraktikern oder Familienangehörigen und Freunden hilfreich, aber unachtsam formulierte - Vorschriften und Regeln beeinträchtigt oder verhindert werden: Dies sind Aufrufe zu «weniger Fett», aber auch «mehr Fett»; «mehr Milchprodukte» oder «weniger Milchprodukte»; «keine Süßigkei- 
Tab. 1. Faktoren, die die Kommunikation beim Essen stören können
Die Portionen dürfen nur klein sein.

Der Patient hat eine Geschmacksverschiebung.

Der Patient muss Diät halten.

Es sind Medikamenteninteraktionen zu beachten.

Es kommt zu früher Sättigung.

Es besteht Appetitverlust.

Es liegt eine Schluckstörung vor.

Der Patient leidet unter Übelkeit.

Der Patient hat ein postoperatives Syndrom.

Es treten Blähungen auf.

ten» oder sogar «keine Kohlenhydrate», aber auch «nur Getreideprodukte»; «mehr Fleisch» oder «keine Wurstwaren»; «mehr Fisch» oder «nur vegetarisch». Selbstverständlich können spezifische Ernährungsvorschriften im Einzelfall durchaus sinnvoll sein, es bleibt jedoch jeweils abzuwägen, in welchem Ausmaß durch die Vorschrift emotionale Genussaspekte betroffen sind und möglicherweise den mutmaßlichen Nutzen der Empfehlung reduzieren.

\section{Die soziale Komponente}

Mahlzeiten sind seit jeher wichtige Bühnen für sozialen Austausch und die Zusicherung von Gemeinsamkeiten und Geborgenheit. Ein faszinierendes Tierexperiment zeigte vor kurzem beim Vergleich mit Kontrolltieren ein reduziertes Tumorwachstum bei Mäusen, die in einem stimulierenden Umfeld gehalten wurden [17]; interessanterweise wird spekuliert, dass neben der hypothalamischen Aktivität endokrine metabolische Mediatoren wie Leptin und Adiponektin an der Vermittlung der Wirkung beteiligt sind. Umfangreiche Daten belegen positive Wirkungen psychosozialer Interventionen auf die Lebensqualität von Tumorpatienten [18]. Wenn auch bisher eindeutige Belege fehlen, so legen mehrere Untersuchungen doch Verbindungen zwischen psychologischen Faktoren wie Stress, Depression und sozialer Isolation und Tumorprogression nahe $[18,19]$.

Kommunikation und soziale Vernetzung im Rahmen von Mahlzeiten mindern Distress, fördern Zuversicht und damit Lebensqualität und Leistungsfähigkeit. Diese Aspekte sollten deshalb in die Betreuung onkologischer Patienten einbezogen werden. Dies umso mehr, da Geschmacksverschiebungen, Appetitlosigkeit, frühe Sättigung, Schluckstörungen und weitere Beeinträchtigungen (Tab. 1) eine ungezwungene Teilnahme eines Tumorkranken an gemeinsamen Mahlzeiten erschweren oder verhindern können. Andere Teilnehmer der Mahlzeit können verunsichert, am Genuss gehindert und abgestoßen werden. Die psycho-sozialen Auswirkungen der so gestörten Interaktionen könnten die Lebensqualität der Beteiligten und möglicherweise sogar Therapieeffekte ungünstig beeinflussen.

\section{Die kognitive Komponente}

Das von Antonovsky postulierte «Kohärenzgefühl», das zur Mobilisierung innerer Kräfte gegen pathologische Faktoren befähigt (Salutogenese), basiert auf dem Bewusstsein ausreichend verfügbarer Ressourcen, um ein lohnendes Ziel auf einem erkennbaren Weg erklärbarer Handlungen und Wirkungen zu erreichen [20]. Ähnlich beschreibt der neuere Begriff der «Patientenkompetenz» die aktive Rolle, die ein Patient in seiner Erkrankung spielen will [21]. Dies gelingt z.B. durch Akzeptanz eines präformierten Gedanken- und Erklärungsgebäudes, das neben dem Ziel einer Erkrankungsverbesserung oder sogar Heilung auch die im täglichen Leben umsetzbaren Schritte zur Zielerreichung vorgibt und erläutert. Auf diesem Wege versprechen Krebsdiäten Lösungen zum Umgang mit und zur Bezwingung von Krebserkrankungen [22]. Kognitive Prozesse ermöglichen so durch Strukturierung von Nahrungsauswahl, Essmenge und Essrhythmus das Gefühl der Kontrolle über einen Teil der Lebensabläufe in dem existenziell bedrohlichen Schatten einer Krebserkrankung. Der Kontrollgewinn wird ermöglicht durch das Auswählen und Einhalten definierter Diätformen, Ernährungsregeln, -vorschriften und -verbote. Kontrollverlust droht dagegen in den gleichen Situationen durch den erkrankungs- oder therapiebedingten Verlust von Leistungsfähigkeit und Konzentration, wenn der Aufwand zur Einhaltung einer - vom Betroffenen für lebenswichtig empfundenen - Diät nicht mehr aufrechterhalten werden kann, durch gastrointestinale Unverträglichkeiten oder wenn der ansonsten vertrauensvoll akzeptierte ärztliche Behandler das Diätkonzept bagatellisiert oder autoritär verwirft. Die hier aufgeführten Argumente sind kein Plädoyer für Krebsdiäten, sie erinnern jedoch daran, dass neben den biochemischen Wirkungen einer Kost andere Aspekte der Kostform für den Patienten eine relevante Bedeutung haben können, die nicht unachtsam übersehen werden sollte.

\section{Die biochemische Komponente}

Neben der Einbindung der Ernährung in psychologische Prozesse auf der Ebene des Individuums steht das enorm weite Gebiet der biochemischen Interaktionen zwischen Nahrungsstoffen, Körperressourcen und physiologischen Prozessen. Nahrungsstoffe dienen dabei als Substrate, d.h. als Bausteine und Energieträger, und induzieren parallel metabolische Signale mit Einfluss auf die Substratnutzung und -verteilung sowie auf den Ablauf von Organ- und Körperfunktionen. Um dem Rechnung zu tragen, empfehlen europäische und amerikanische Leitlinien zur Ernährung onkologischer Patienten bei jedem Patientenkontakt eine kurze Erhebung zur Ernährungssituation [23, 24]. Eine solche Kurzerfassung sollte sich auf Appetit, Nahrungsaufnahme, Gewichtsverlauf und inflammatorische Stoffwechselveränderungen konzentrieren.

$\mathrm{Zu}$ den typischen Charakteristika maligner Zellen werden heute metabolische Muster gerechnet [25]; dazu gehören die durch lokale Hypoxie induzierte Aktivierung der HIF (hypo- 
xia-inducible factors), der Neoangiogenese (unter anderem via VEGF, vascular endothelial growth factor), der Serin-/ Threonin-Kinase Akt/PKB, von Defekten in Mitochondrienfunktionen und der oxidativen Phosphorylierung sowie der gesteigerten Expression von Glukosetransportern GLUT1 (typisch nutzbar für ${ }^{18} \mathrm{~F}$-Fluordesoxyglukose-Positronenemissionstomographie (18-FDG-PET)) sowie eine ungebremste Glykolyse zu Laktat. Bereits 1924 postulierte Warburg, dass durch Hypoxie induzierte Stoffwechselveränderungen zur Tumorentstehung und zum malignen Phänotyp mit erhöhter Glykolysekapazität beitragen und diese Zellen anfällig für einen Glukoseentzug sein müssen [26]. Interessanterweise ließ sich in vitro zeigen, dass eine durch Transfektion induzierte Aktivierung von Akt/PKB das Zellüberleben in glukosefreiem Medium einschränkt [27], sodass die auf Warburgs Theorien gründenden Konzepte einer antitumoralen Wirkung glukosefreier Kost neuen Auftrieb erhielten [22, 28]. Bisher liegen allerdings keine überzeugenden klinischen Daten vor, die solche Ernährungskonzepte als antitumorale Maßnahme stützen könnten.

Bei fortgeschrittener Tumorerkrankung entwickelt sich häufig eine systemische Inflammationsreaktion [29-31]. Die resultierende Insulinresistenz mindert bei Patienten mit Tumorkachexie die Glukosetoleranz, während die Verwertbarkeit von Fett nicht beeinträchtigt ist [32]. Dies wird als Argument für klinische Vorteile einer eher fettbetonten Kost bei Tumorpatienten gesehen [33]. Da die metabolischen Veränderungen an der Genese der Tumorkachexie beteiligt zu sein scheinen, werden seit über 10 Jahren anti-inflammatorische Ansätze geprüft, unter Einsatz langkettiger Fettsäuren der Omega-3-Reihe (Eicosapentaensäure, EPA) als kompetitive Antagonisten des pro-inflammatorischen Precursors Omega6-Arachidonsäure. Ein systematischer Review zu 17 kontrollierten, aber nicht durchgehend randomisierten Studien bei Patienten mit Tumorkachexie kam 2007 zu dem Ergebnis, dass EPA in Dosen von mindestens 1,5 g/Tag den Appetit, das Körpergewicht und die Lebensqualität bessert [34]. Eine randomisierte aktuelle Studie zeigte unter $2 \mathrm{~g} / \mathrm{Tag}$ EPA eine Besserung der Energieaufnahme, des Körpergewichts und der fettfreien Körpermasse [35]. 2 weitere, nichtrandomisierte vergleichende Untersuchungen ergaben bei Patienten mit Bronchialkarzinom für die Einnahme von 2,5 g/Tag Omega-3Fettsäuren eine signifikante Verbesserung sowohl des Ernährungszustands als auch des Tumoransprechens auf eine Chemotherapie (60\% vs. $20 \%)$ und des 1-Jahres-Überlebens $(60 \%$ vs. $39 \%)[36,37]$.

\section{Fazit}

Die Bedeutung der Nahrungsaufnahme kann unter verschiedenen Aspekten gesehen werden. Sie kann eine Vielzahl körperlicher sowie psychologischer Parameter beeinflussen. Diese unterschiedlichen Aspekte sollten bei jedem Patienten angesprochen werden; die emotionalen, kommunikativen und kognitiven Kontrollaspekte sollten ernst genommen und nicht mit voreilig einseitigen Urteilen bagatellisiert werden. Wenn erforderlich, sollten individuelle Problemlösungen gesucht und der Betroffene professionell unterstützt werden. Die ausreichende Versorgung mit Nährstoffen sowie die metabolische Stoffwechsellage sollten parallel zu jeder Tumortherapie beachtet und in das Therapiekonzept integriert werden

\section{Gleichklang von Körper und Seele: Die Mind-Body- Medizin Verantwortliche Autorin: Anna Paul, Essen}

Die psychische Unterstützung von Krebspatienten durch Förderung von Achtsamkeit und Selbstfürsorge ist ein wesentlicher Bestandteil der Integrativen Onkologie und eine Domäne der Mind-Body-Medizin. Es handelt sich dabei um einen naturheilkundlichen Behandlungsansatz aus dem Bereich der Ordnungstherapie. Die US-amerikanischen National Institutes of Health (NIH) definieren die Mind-Body-Medizin als ein Verfahren, «das Interaktionen zwischen Gehirn, Psyche, Körper und Verhalten berücksichtigt und die direkte gesundheitsfördernde Wirkung bestimmter emotionaler, mentaler, sozialer, spiritueller und verhaltensabhängiger Einflussfaktoren nutzt». Es geht darum, die Fähigkeit eines Menschen zur Selbsterkenntnis und Selbstfürsorge zu respektieren und daraus entsprechende Therapieansätze abzuleiten, mit dem Ziel einen gesundheitsförderlichen Lebensstil zu entwickeln. Die Mind-Body-Medizin gibt Antworten auf die von Krebspatienten häufig gestellte Frage: «Was kann ich selbst für mich tun?»

Die Techniken der Mind-Body-Medizin (Abb. 3) zielen auf die Optimierung der Selbsthilfe und eine Stärkung der Selbstheilungskräfte ab. Neben klassischen Interventionsmaßnahmen wie Ernährungsberatung, Bewegungsanleitung und Hilfe in sozialen Fragen nehmen Techniken zur Stressbewältigung einen großen Raum ein. Die verschiedenen Verfahren werden je nach Bedarf kombiniert, wobei das von der Harvard Medical School entwickelte «Cancer Program» sowie das ebenfalls aus den USA stammende Verfahren zur MBSR (mindfulness-based stress reduction) die Eckpfeiler bilden.

Therapieangebote in der Mind-Body-Medizin sind variabel. Es gibt Einzelinterventionen wie auch Programme von unterschiedlicher Dauer (ab 6 Wochen bis zu etwa 1 Jahr), die bei verschiedenen Krebserkrankungen eingesetzt werden können. Der Nutzen dieser Verfahren zeigte sich unter anderem bei Männern mit Prostatakarzinom, die an einem auf der Mind-Body-Medizin basierten Programm teilgenommen hatten. Unter aktiver Anleitung wurden Lebensstiländerungen durch Fokussierung auf eine vegane, fettarme Ernährung, ein moderates Bewegungstraining, Maßnahmen zur Stressbewältigung und intensive Gruppensitzungen umgesetzt. Die Interventionen führten unter anderem zu rückläufigen Prostata-spezifischen Antigen(PSA)-Werten, einem Anstieg der 


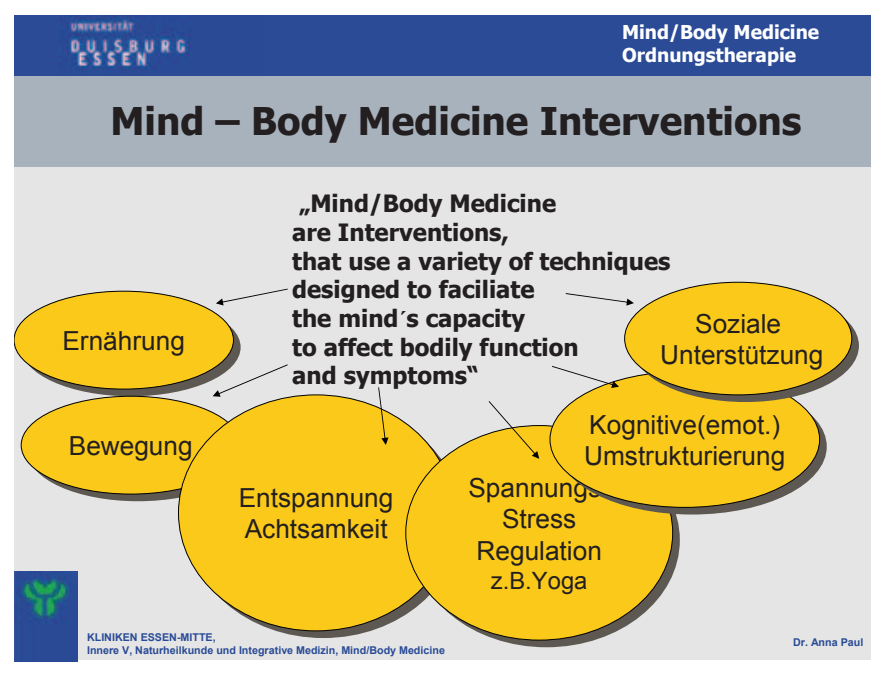

Abb. 3. Elemente der Mind-Body-Medizin.

Lebensqualität sowie einer Besserung der Sexualfunktionen [38].

Die theoretischen Grundlagen der Mind-Body-Medizin orientieren sich am naturheilkundlichen Ansatz der Salutogenese, die die Ressourcen des Patienten und nicht seine krankheitsbedingten Beeinträchtigungen in den Mittelpunkt stellt. Des Weiteren fließen Erkenntnisse der Stressforschung, der Psychoneuroimmunologie und der Stressverarbeitung (Relaxationsresponse) in die Programme ein [39]. Auch Übungen zur Achtsamkeit, Anleitungen zu Lebensstiländerungen und die Arbeit mit sozialkognitiven Verhaltensänderungsmodellen gehören dazu. Voraussetzung für eine erfolgreiche Umsetzung des Mind-Body-Konzeptes mit nachhaltiger Veränderung des Lebensstils ist das Zusammenspiel von Information, Training und Motivation des Patienten.

Ziel der Mind-Body-Medizin ist das Erlangen einer sogenannten «stress hardiness» (Bewältigungsfähigkeit), etwa durch Anwendung von Entspannungsmethoden. So hat eine Studie gezeigt, dass mit Yoga-Techniken das Stressempfinden innerhalb von nur 3 Monaten signifikant gesenkt werden kann [40]. Eine ausgeprägte Achtsamkeit ist Basis für Selbstwahrnehmung und Selbstfürsorge. Sie hilft bei der Verarbeitung der Krebsdiagnose (Schuldfrage) und beim Umgang mit Zukunftsängsten. In 8-wöchigen MBSR-Kursen, die unter anderem die Vermittlung von Yoga- und Meditationstechniken zum Inhalt haben, kann die Achtsamkeit geschult werden. Solche Programme können zu einer Verbesserung der psychischen Befindlichkeit (positive Grundstimmung) und einer Stärkung der Immunfunktionen führen, wie bei gesunden Probanden gezeigt wurde [41].

Die Änderung von ungünstigen Verhaltensweisen - ein weiteres Ziel der Mind-Body-Medizin - kann viel Zeit beanspruchen und hängt auch davon ab, in welchem Umfang der Patient Änderungen seines Lebensstils akzeptiert. Unsere Arbeitsgruppe arbeitet dabei nach dem transtheoretischen Mo-

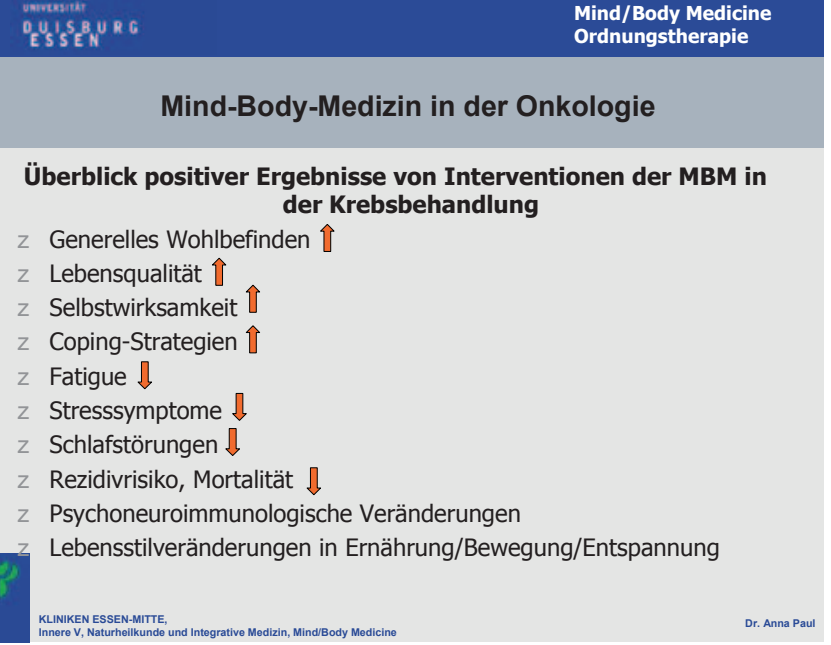

Abb. 4. Mind-Body-Medizin in der Onkologie.

dell [42], wonach Verhaltensänderungen verschiedene Stadien von unterschiedlicher Dauer durchlaufen müssen, bevor sie dauerhaft implementiert werden.

Mit den Techniken der Mind-Body-Medizin können zahlreiche positive Wirkungen hervorgerufen werden, von denen auch Krebspatienten profitieren (Abb. 4). Diese Effekte sind in Studien unterschiedlicher Qualität belegt, besonders umfangreich ist das Datenmaterial zur MBSR. In Übersichtsarbeiten und Metaanalysen [43-45] fanden sich teils große Effektstärken bei der Reduktion von Stress und Angst sowie Hinweise, dass sich durch entsprechende Schulung der Achtsamkeit auch die Depressivität von Patienten bessert. Ähnlich gut belegt sind die Effekte von Yoga [46-48], mit dem sich ebenfalls Angst, Depression und Stress reduzieren lassen und das sich auch bei Fatigue und Schlafproblemen als hilfreich erwiesen hat.

Das von unserer Klinik angebotene Mind-Body-Programm umfasst 6 Kursstunden pro Woche über eine Dauer von 11 Wochen («Essener Modell»; Abb. 5). Ähnliche Angebote gibt es an einigen anderen naturheilkundlichen Kliniken, außerdem haben sich zahlreiche MBSR-Gruppen gebildet, die über die Homepage www.mbsr-verband.org zu erreichen sind.

\section{Der Beitrag der traditionellen asiatischen Medizin zur Integrativen Onkologie \\ Verantwortlicher Autor: Hans Lampe, Rostock}

Unter der traditionellen asiatischen Medizin werden die klassische chinesische Medizin (TCM), die indische Medizin (Ayurveda) und damit verwandte Therapien (koreanische, tibetische und japanische Medizin) zusammengefasst. Während in den westlichen Medizinsystemen die morphologische Diagnostik und insbesondere die quantitative Messung entscheidend für Diagnose und Therapie sind, liegt der Schwerpunkt 


\section{Integrative Onkologie Mind-Body-Medizin "Essener Modell“}

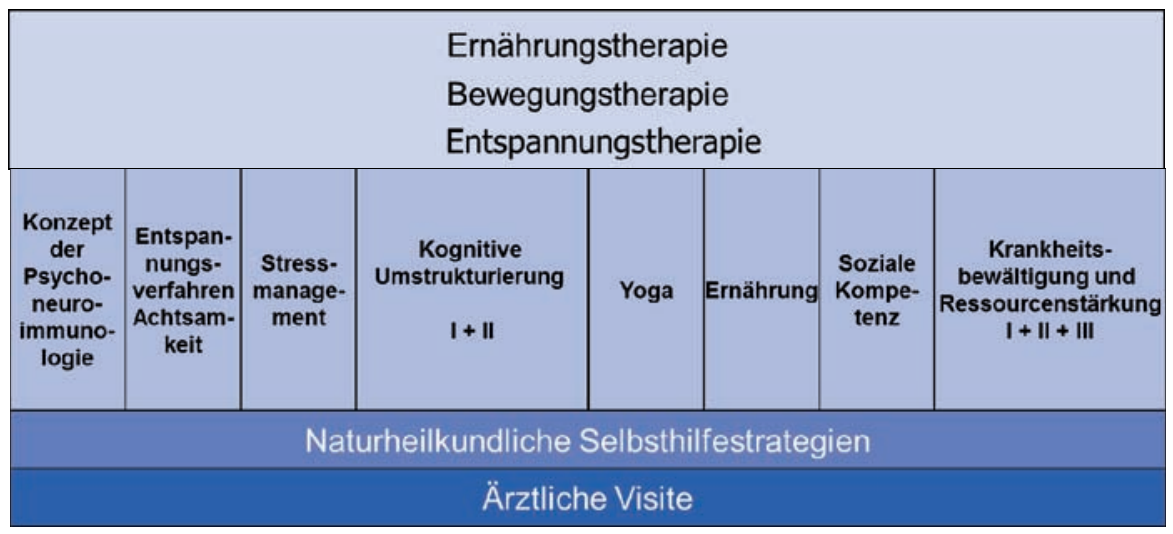

Abb. 5. Therapieangebote der Mind-BodyMedizin an den Kliniken Essen.

\section{Ärztliche Visite}

\section{Pharmakologische Therapie in West und} Ost

Westliche Medizin
- Isolierte Monosubst.
- Angriffspunkt:
Tumorzelle
- Wirkung: schnell
- Problem: Rückfälle
- Perspektiven:
bessere Spezifität
neue Ansatzpunkte

\section{Asiatische Medizin}

- Komplexe Rezeptur

- Angriffspunkt: Gleichgewicht

- Wirkung: langsam

- Problem: Langsamkeit

- Perspektiven:

„Stabilisierung“ in einem kombinierten Konzept

Abb. 6. Unterschiede in der Pharmakotherapie.

der östlichen Medizin auf den funktionellen Abläufen (Bewegungsrichtungen), für deren Beurteilung die Erhebung qualitativer Parameter entscheidend ist. Das Verständnis für die asiatische Medizin als eigenständiges System erfordert deshalb ein gewisses Umdenken. Eine andere Möglichkeit besteht darin, Extrakte aus der asiatischen Medizin - etwa einzelne Inhaltstoffe - in das westliche System einzugliedern. So haben z.B. Auskochungen (Dekokte) bestimmter pflanzlicher und tierischer Substanzen eine antikanzerogene Wirkung. Aus Dekokten isolierte Monosubstanzen wie das Indirubin wurden zu modernen Krebsmedikamenten. Auch die Camptothecin-Derivate (Irinotecan, Topotecan) gehören zum Fundus der chinesischen Medizin und werden heute gegen verschiedene solide Tumoren eingesetzt.

Häufig lässt sich nur schwer ermitteln, welche Wirkung den einzelnen Inhaltstoffen eines Dekoktes zuzuordnen ist, zumal sich durch das Auskochen der Pflanzen bzw. Pflanzengemische weitere pharmakologisch relevante Wirkungen er- geben. Studien konzentrieren sich zumeist auf die Wirkung einzelner Arzneipflanzen. So scheint Radix Astragali (Huang Qi) die Wirksamkeit einer platinbasierten Chemotherapie bei fortgeschrittenem nichtkleinzelligem Lungenkrebs (NSCLC) zu verbessern, wie eine Metaanalyse randomisierter Studien gezeigt hat [49]. Eine Gesamtbeurteilung ist allerdings schwierig, weil die Einzelstudien klein waren und sowohl die PlatinRegime als auch die Pflanzenzubereitungen nicht direkt vergleichbar waren. Für die Kombination der 2 Medizinsysteme gibt es keine langfristigen Erfahrungen, hier besteht Forschungsbedarf. Dies gilt auch für eine Studie, deren Originaltext nur in Chinesisch vorliegt [50] und somit für europäische Ärzte nur in Zusammenfassungen erhältlich ist. Die Autoren dieser Arbeit wiesen für die Kombination aus traditioneller chinesischer Medizin und westlicher Medizin eine verbesserte Wirksamkeit (Überleben) bei Patienten mit chirurgisch reseziertem Kolorektalkarzinom nach. Die Kombination der beiden Verfahren war effektiver als die alleinige Anwendung des Standards an adjuvanten Therapien (Chemo- und/oder Radiotherapie).

Wer sich nicht nur für die Arzneistoffe als Einzelkomponenten der östlichen Medizin interessiert, sondern sich mit dem System als Ganzes auseinandersetzen will, sollte die in diesem Gedankengebäude vorherrschende qualitative Beschreibung von Abläufen nachvollziehen können. Sie kann komplementär die quantitative Messung ergänzen, anhand derer wir in der westlichen Medizin die Abweichung von der Norm erfassen. Wissenschaftstheoretisch werden westliche Mediziner bei einer Erkrankung zunächst eine Ursachenanalyse vornehmen (kausale Analyse), während chinesische Ärzte nach der derzeitigen Abweichung vom gesunden Lebensverlauf und deren Beeinflussung (induktive Synthese) fragen.

Ziel der traditionellen asiatischen Medizin ist nicht eine spezifische Tumorbehandlung, sondern den erkrankten Orga- 


\section{Integration in bestehende Behandlungskonzepte}

\section{Einsatzmöglichkeiten der TCM}

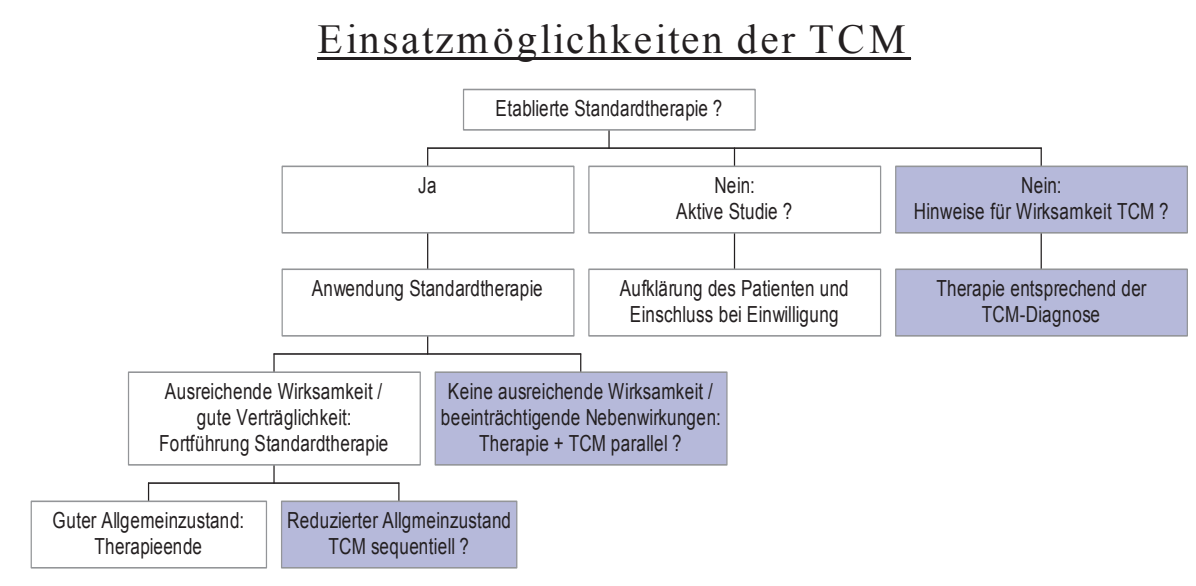

Abb. 7. Integration der TCM in westliche Therapiekonzepte.

Tab. 2. Therapieschen Medizin ansätze in der asiati-

\begin{tabular}{l} 
Pharmakologische Therapie \\
Pflanzliche, tierische, mineralische Extrakte \\
Akupunktur/Akupressur \\
Diätetik \\
TuiNa \\
Qigong/Taijiquan/Yoga \\
Ölbehandlungen \\
Schwitzkuren \\
\hline
\end{tabular}

Pharmakologische Therapie

Pflanzliche, tierische, mineralische Extrakte

Qigong/Taijiquan/Yoga

Schwitzkuren

nismus wieder in ein Gleichgewicht zu bringen, die Auseinandersetzung des Körpers mit der Erkrankung zu beeinflussen. Die chinesische Medizin nutzt zur Diagnose Funktionskreise und Leitkriterien, mit denen sich der aktuelle Körperzustand beschreiben lässt und sich krankhafte Abweichungen erfassen lassen. Die zugehörigen Therapiemethoden sind vielfältig (Tab. 2), wobei pharmakologische Therapien mit Naturstoffen dominieren. Daraus ergeben sich Unterschiede und mögliche Ergänzungen zur westlichen Medizin (Abb. 6).
Die traditionelle asiatische Medizin hat bisher abgesehen von Monosubstanzen wenig Eingang in die westliche Medizin gefunden. Womöglich nehmen wir das Potenzial dieser Therapieform nicht ausreichend wahr, zumal wir noch wenig über die Pharmakologie der eingesetzten Dekokte wissen. Es wäre sinnvoll, die Chancen einer komplementären Anwendung auszuloten und mögliche neue Therapieansätze (Zelldifferenzierung, Anti-Angiogenese, Beeinflussung des Microenvironments) zu erforschen. Einzelne Verfahren der chinesischen Medizin sind bereits in konventionelle Therapiekonzepte integriert, wie es in unserer Klinik der Fall ist (Abb. 7). Dabei haben sich die östlichen Verfahren insbesondere zur Behandlung unerwünschter Wirkungen von Standardtherapien als hilfreich erwiesen.

\section{Disclosure Statement}

Es bestehen keine Interessenkonflikte.

\section{Literatur}

1 Schüle K: The rank value of sports and movement therapy in patients with breast or pelvic cancer. Rehabilitation 1983;22:36-39.

2 Holmes MD, et al.: Physical activity and survival after breast cancer diagnosis. JAMA 2005;293:24792486.

3 Kenfield SA, et al.: Physical activity and survival after prostate cancer diagnosis in the Health Professionals Follow-Up Study. J Clin Oncol 2011;29: 726-732.

4 Windsor PM, et al.: A randomized, controlled trial of aerobic exercise for treatment-related fatigue in men receiving radical external beam radiotherapy for localized prostate carcinoma. Cancer 2004;101:550-557.

$\checkmark 5$ Monga U, et al.: Exercise prevents fatigue and improves quality of life in prostate cancer patients undergoing radiotherapy. Arch Phys Med Rehabil 2007;88:1416-1422.
6 Segal RJ, et al.: Resistance exercise in men receiving androgen deprivation therapy for prostate cancer. J Clin Oncol 2003;21:1653-1659.

7 Baumann FT, et al.: Physical activity for patients undergoing an allogeneic hematopoietic stem cell transplantation: benefits of a moderate exercise intervention. Eur J Haematol 2011;87:148-156.

8 Baumann FT, et al.: A controlled randomized study examining the effects of exercise therapy on patients undergoing haematopoietic stem cell transplantation. Bone Marrow Transplant 2010;45:355-362.

9 Schmitz KH, et al.: Weight lifting in women with breast-cancer-related lymphedema. N Engl J Med 2009;361:664-673.

10 Nieman DC: Immune response to heavy exertion. J Appl Physiol 1997;82:385-394.
1 Overgård $\mathrm{M}$, et al.: Does physiotherapist-guided pelvic floor muscle training reduce urinary incontinence after radical prostatectomy? A randomised controlled trial. Eur Urol 2008;54:438-448.

12 Centemero A, et al.: Preoperative pelvic floor muscle exercise for early continence after radical prostatectomy: a randomised controlled study. Eur Urol 2010;57:1039-1043.

13 Baumann F, Bloch W, Jäger E (Hrsg): Sport und körperliche Aktivität in der Onkologie. Berlin, Heidelberg, Springer, 2012.

14 Schmitz KH, et al.: American College of Sports Medicine roundtable on exercise guidelines for cancer survivors. Med Sci Sports Exerc 2010;42:14091026; Erratum in: Med Sci Sports Exerc 2011;43:195.

15 Capaldi ED: Why We Eat What We Eat: The Psychology of Eating. Washington, American Psychological Association, 2001. 
16 Hopkinson JB: The emotional aspects of cancer anorexia. Curr Opin Support Palliat Care 2010;4: 254-258.

17 Cao L, et al.: Environmental and genetic activation of a brain-adipocyte BDNF/leptin axis causes cancer remission and inhibition. Cell 2010;142:52-64.

18 Lutgendorf SK, et al.: Host factors and cancer progression: biobehavioral signaling pathways and interventions. J Clin Oncol 2010;28:4094-4099.

19 Lutgendorf SK, Sood AK: Biobehavioral factors and cancer progression: physiological pathways and mechanisms. Psychosom Med 2011;73:724-730.

20 Antonovsky A: Health, Stress and Coping, ed 1. San Francisco, Jossey-Bass Inc Pub, 1979.

21 Nagel GA: Patientenkompetenz. Krankenhauspharmazie 2005;26:128-133.

22 Arends J: Gibt es eine Krebsdiät. Forum 2011;26: 25-29.

23 Bozzetti F, et al.: ESPEN guidelines on parenteral nutrition: non-surgical oncology. Clin Nutr 2009;28: 445-454.

24 August DA, et al.: A.S.P.E.N. clinical guidelines: nutrition support therapy during adult anticancer treatment and in hematopoietic cell transplantation. JPEN J Parenter Enteral Nutr 2009;33:472500.

25 Hanahan D, Weinberg RA: Hallmarks of cancer: the next generation. Cell 2011;144:646-674.

26 Warburg O, et al.: Über den Stoffwechsel der Carzinomzelle. Biochem Z 1924;152:309-344.

27 Elstrom RL, et al.: Akt stimulates aerobic glycolysis in cancer cells. Cancer Res 2004;64:3892-3899.

28 Arends J: Maligne Tumoren - Transketolase-like 1 (TKTL 1) - Ketogene Diät. Aktuel Ernahrungsmed 2008;33:80-81.

29 Dvorak HF: Tumors: wounds that do not heal. Similarities between tumor stroma generation and wound healing. N Engl J Med 1986;315:1650-1659.
30 Deans C, Wigmore SJ: Systemic inflammation, cachexia and prognosis in patients with cancer. Curr Opin Clin Nutr Metab Care 2005;8:265-269.

31 Mantovani A, et al.: Cancer-related inflammation. Nature 2008;454:436-444.

32 Körber J, et al.: Increased lipid utilization in weight losing and weight stable cancer patients with normal body weight. Eur J Clin Nutr 1999;53:740-745.

33 Holm E, Kämmerer U: Fette und Kohlenhydrate in Ernährungskonzepten für Tumorpatienten. Aktuel Ernahrungsmed 2011;36:286-298.

34 Colomer R, et al.: N-3 fatty acids, cancer and cachexia: a systematic review of the literature. Br J Nutr 2007;97:823-831.

35 van der Meij BS, et al.: Oral nutritional supplements containing (n-3) polyunsaturated fatty acids affect the nutritional status of patients with stage III non-small cell lung cancer during multimodality treatment. J Nutr 2010;140:1774-1780.

36 Murphy RA, et al.: Nutritional intervention with fish oil provides a benefit over standard of care for weight and skeletal muscle mass in patients with nonsmall cell lung cancer receiving chemotherapy. Cancer 2011;117:1775-1782.

37 Murphy RA, et al.: Supplementation with fish oil increases first-line chemotherapy efficacy in patients with advanced nonsmall cell lung cancer. Cancer 2011;117:3774-3780.

38 Daubenmier JJ, et al.: Lifestyle and health-related quality of life of men with prostate cancer managed with active surveillance. Urology 2006;67:125-130.

39 Dobos G, Kümmel S: Gemeinsam gegen Krebs. München, Verlag Zabert Sandmann, 2011.

40 Michalsen A, et al.: Rapid stress reduction and anxiolysis among distressed women as a consequence of a three-month intensive yoga program. Med Sci Monit 2005;11:CR555-CR561.
1 Davidson RJ, et al.: Alterations in brain and immune function produced by mindfulness meditation. Psychosom Med 2003;65:564-570.

42 Prochaska JO, DiClemente CC: Self change processes, self efficacy and decisional balance across five stages of smoking cessation. Prog Clin Biol Res 1984:156:131-140.

43 Matchim Y, et al.: Mindfulness-based stress reduction among breast cancer survivors: a literature review and discussion. Oncol Nurs Forum 2011;38: E61-E71.

44 Shennan C, et al.: What is the evidence for the use of mindfulness-based interventions in cancer care? A review. Psychooncology 2011;20:681-697.

45 Cramer $\mathrm{H}$, et al.: Can yoga improve fatigue in breast cancer patients? A systematic review. Acta Oncol 2012;51:559-560.

46 Smith KB, Pukall CF: An evidence-based review of yoga as a complementary intervention for patients with cancer. Psychooncology 2009;18:465-475.

47 Lin KY, et al.: Effects of yoga on psychological health, quality of life, and physical health of patients with cancer: a meta-analysis. Evid Based Complement Alternat Med 2011;2011:659876.

48 Cramer H, et al.: Can yoga improve fatigue in breast cancer patients? A systematic review. Acta Oncol 2012;51:559-560.

49 McCulloch M, et al.: Astragalus-based Chinese herbs and platinum-based chemotherapy for advanced non-small-cell lung cancer: meta-analysis of randomized trials. J Clin Oncol 2006;24:419-430.

50 Yang YF, et al.: Cohort study on the effect of a combined treatment of traditional Chinese medicine and Western medicine on the relapse and metastasis of 222 patients with stage II and III colorectal cancer after radical operation. Chin J Integr Med 2008;14:251-256. 